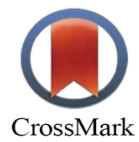

\title{
A Large Intracolonic Mass in a Patient with Peutz-Jeghers Syndrome
}

\author{
Ran Wang ${ }^{1,2}$, Xingshun $\mathrm{Qi}^{1{ }^{1} \text {, }}$, Xiaodong Shao ${ }^{1}$, Xiaozhong Guo ${ }^{1}$
}

1. Department of Gastroenterology, General Hospital of the Shenyang Military Area, Shenyang, China.

2. Postgraduate College, Liaoning University of Traditional Chinese Medicine, Shenyang, China.

\footnotetext{
* Corresponding Author:

Xingshun Qi, MD

Department of Gastroenterology, General Hospital of the Shenyang Military Area, Shenyang, China.

Tel: +862428897603

fax: + 862428851113

Email: xingshunqi@126.com
}

Received: 18 Nov. 2016

Accepted: 12 Feb. 2017

\section{ABSTRACT}

Peutz-Jeghers syndrome (PJS) is characterized by scattered black pigmentations on fingers and lips and multiple polyps in the gastrointestinal tract. Patients with PJS often have severe complications secondary to multiple large polyps. Herein, we present a young woman complicated with a large polyp in her colon without any clinical symptoms. We also emphasized the necessity of early diagnosis and treatment of multiple polyps in such patients.

\section{KEYWORDS:}

Peutz-Jeghers syndrome, Colonic endoscopy, Polyp, Pigmentation.

Please cite this paper as:

Wang R, Qi X, Shao X, Guo X. A Large Intracolonic Mass in a Patient with Peutz-Jeghers Syndrome. Middle East J Dig Dis 2017;9:173-175. DOI: 10.15171/mejdd.2017.70.

\section{INTRODUCTION}

Peutz-Jeghers syndrome (PJS) is a familial inherited disease characterized by scattered black pigmentations on fingers and lips and multiple polyps in the gastrointestinal tract. In 1921, Dr. Peutz described a family with multiple polyps and scattered mucocutaneous pigmentations and recognized the inherited nature of this disease. In 1949, Jeghers and colleagues noted the inner-connection between pigmentations and multiple gastrointestinal polyps. It is reported that the morbidity of PJS ranges from 1:50,000 to 1:200,000. ${ }^{1}$ Patients with PJS may be complicated with anemia, diarrhea, abdominal pain, intestinal obstruction, and intussusception. Some complications, including intestinal obstruction, and intussusception, are potentially life-threatening. PJS also carries a high risk of malignancy. Early diagnosis and endoscopic screening are important in patients with PJS. Herein, we reported an interesting case of PJS complicated with a large polyp in the colon without any other clinical symptoms.

\section{CASE REPORT}

A 24-year-old woman referred to our hospital for endoscopic examinations. She had scattered pigmentations on fingers and lips since she was 5 years old. She had received laser therapy for these pigmentations in June 2013, October 2013, and March 2013. Her cosmetologist advised her to undergo a colonoscopy. No history of other diseases was recorded. Her father and younger brother had no similar complications. But her mother also had black pigments on her fingers and lips 


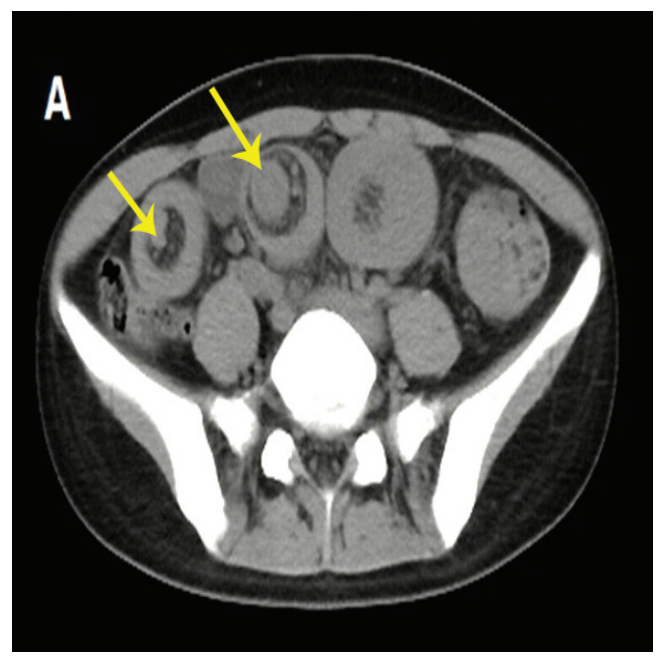

Fig.A: Axial computed tomography showing multiple polyps all over colon (yellow arrow).

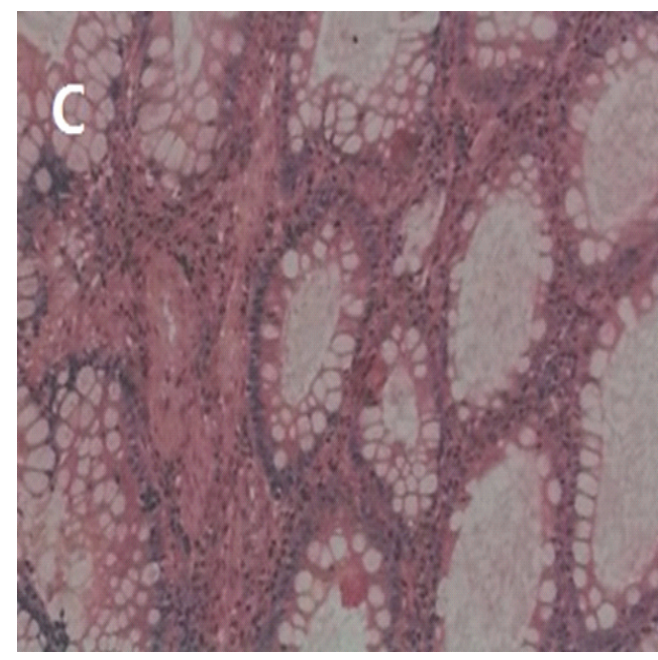

Fig.C: Histological findings suggesting hamartomatous polyps with dysplasia.

without any endoscopic examinations. Her mother eventually died of cholangiocarcinoma. The abdomen was not tender on physical examinations. Laboratory tests showed that red blood cell count was $4.77 \times 10^{12} / \mathrm{L}$, hemoglobin level was $100 \mathrm{~g} / \mathrm{L}$, and albumin level was $33.8 \mathrm{~g} / \mathrm{L}$. Other laboratory results were normal. Abdominopelvic computed tomography showed numerous polyps all over her colon (figure A). The largest polyp almost occupied the whole colon cavity. An endoscopic resection of polyps was performed. The diameter of the largest polyp was 5 centimeters (figure B). Histological

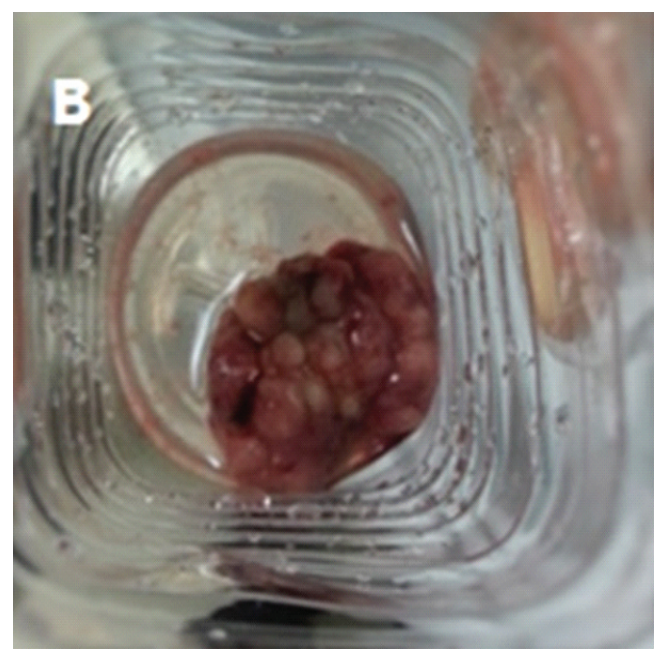

Fig.B: The largest polyp was endoscopically resected.

evaluation suggested hamartomatous polyps with dysplasia. Immunohistochemistry staining test showed the following: CK-P $(+)$, CEA $(+)$, EMA $(+)$, CK8/18 (+), CDX-2 (+), CerbB-2 (1+), Syn (-), Villin (+), Ki67 (+) 30\%, and P53 (+) 60\% (figure $\mathrm{C}$ ). She is being followed up without any other complaints.

\section{DISCUSSION}

There is still some controversy on the malignant potential of hamartomatous polyps. Some studies suggest that PJS polyps have a high risk of malignancy. ${ }^{1}$ However, others suggest that PJS is an epiphenomenon preceding a cancer-prone state. ${ }^{2}$ Our previous case report demonstrated a family of four patients diagnosed as having PJS, of whom three had hamartomatous polyps and one had inflammatory polyps. ${ }^{3}$ Notably, one of them died of lung adenocarcinoma. Similarly, our patient's mother presented with black pigments on fingers and lips, and then died of cholangiocarcinoma. Thus, PJS might follow the hamartoma-carcinoma sequence.

Treatment selection for PJS mainly depends on its related complications. Endoscopic screening should be performed every 2 years, ${ }^{4}$ and endoscopic resection of polyps can be performed at the same time of the examination, if necessary. Emergency surgery is necessary in the cases of intestinal obstruction and intussusceptions secondary to multiple and/or large polyps.

The present case was not complicated with any gas- 
trointestinal symptoms. However, multiple polyps were found all over the colon. The largest polyp had almost occupied the whole colon cavity. Our patient would have intestinal obstruction or intussusceptions, if she did not undergo polyp resection. Thus, patients with black pigmentations on fingers and lips may regularly undergo

colonic endoscopy. It is also important to introduce the knowledge regarding PJS to cosmetologists. Early diagnosis and endoscopic resection in high-risk patients are needed.

\section{CONFLICT OF INTEREST}

The authors declare no conflict of interest related to this work.

\section{REFERENCES}

1. van Lier MG, Wagner A, Mathus-Vliegen EM, Kuipers EJ, Steyerberg EW, van Leerdam ME. High cancer risk in Peutz-Jeghers syndrome: a systematic review and surveillance recommendations. $\mathrm{Am}$ $J$ Gastroenterol 2010;105:1258-64; author reply 1265. doi:10.1038/ajg.2009.725.

2. Jansen M, de Leng WW, Baas AF, Myoshi H, Mathus-Vliegen L, Taketo MM, et al. Mucosal prolapse in the pathogenesis of Peutz-Jeghers polyposis. Gut 2006;55:1-5. doi:10.1136/gut.2005.069062.

3. Wang R, Qi X, Liu X, Guo X. Peutz-Jeghers syndrome: Four cases in one family. Intractable Rare Dis Res 2016;5:42-3. doi:10.5582/irdr.2015.01036.

4. Higham P, Alawi F, Stoopler ET. Medical management update: Peutz Jeghers syndrome. Oral Surg Oral Med Oral Pathol Oral Radiol Endod 2010;109:5-11. doi:10.1016/j.tripleo.2009.08.022. 